Erratum

\title{
IS research methods: inclusive or exclusive?
}

\section{Allen S Lee}

Journal of Information Technology (2012) 27, 178. doi:10.1057/jit.2012.2

Correction to: Journal of Information Technology (2011) 26, 296-298. doi:10.1057/jit.2011.24

Unfortunately, the published title of the above paper was incomplete. The full and correct title for this commentary should have been:

'Commentary on Davison and Martinsons: Methodological practice and policy for organisationally and socially relevant IS research: an inclusive - exclusive perspective'.

Allen S Lee 